\title{
Erratum: Hard thermal loops, to quadratic order, in the background of a spatial 't Hooft loop \\ [Phys. Rev. D 80, 036004 (2009)]
}

Yoshimasa Hidaka $\odot$ and Robert D. Pisarski

(Q) (Received 2 September 2020; published 28 September 2020)

DOI: 10.1103/PhysRevD.102.059902

1. The right-hand side of Eq. (118) is missing a factor of " $i$ ":

$$
\frac{1}{16 \pi^{2}} \int_{0}^{\infty} d k k^{2}\left(n\left(k-i Q_{1}\right)-n\left(k+i Q_{1}\right)+n\left(k-i Q_{2}\right)-n\left(k+i Q_{2}\right)\right)=i \frac{\pi T^{3}}{12}\left(\mathcal{A}_{0}\left(Q_{1}\right)+\mathcal{A}_{0}\left(Q_{2}\right)\right) .
$$

2. Similarly, there is an " $i$ " missing in Eq. (137),

$$
\tilde{\mathcal{J}}^{\mu \nu}\left(P^{12}, Q_{1}, Q_{2}\right) \stackrel{\mathrm{HTL}}{\approx} i \frac{\pi T^{3}}{6}\left(\mathcal{A}_{0}\left(Q_{1}\right)+\mathcal{A}_{0}\left(Q_{2}\right)\right) \delta \Gamma^{\mu \nu}\left(P^{12}\right)+\frac{T^{2}}{24}\left(\mathcal{A}\left(Q_{1}\right)+\mathcal{A}\left(Q_{2}\right)\right) \delta \Pi^{\mu \nu}\left(P^{12}\right) .
$$

3. Equation (158) has an additional factor of $i \pi / 3$ :

$$
\mathcal{K}^{a b, c d}(Q)=\frac{i \pi}{3} 2 g^{2} T^{3} \delta^{a d} \delta^{b c}\left[\sum_{e=1}^{N_{c}}\left(\mathcal{A}_{0}\left(Q^{a e}\right)+\mathcal{A}_{0}\left(Q^{e b}\right)\right)-N_{f}\left(\mathcal{A}_{0}\left(\tilde{Q}^{a}\right)+\mathcal{A}_{0}\left(-\tilde{Q}^{b}\right)\right)\right] .
$$

